\title{
Resistente Gonokokken breiten sich aus
}

Nach Ländern in Asien und Europa werden nun auch aus Kanada erhöhte Raten an Therapieversagen bei der Behandlung von Gonorrhö-Patienten mit Cefixim gemeldet.

Labormediziner in Toronto analysierten Testergebnisse von 133 Patienten, die wegen einer Infektion mit Neisseria gonorrhoeae mit einmal $400 \mathrm{mg}$ Cefixim oral behandelt worden waren. Der Therapieerfolg wurde nach zwei bis vier Wochen überprüft. Als Therapieversagen galt, wenn nach der Behandlung derselbe
Keim molekularbiologisch nachweisbar war und eine erneute Infektion ausgeschlossen werden konnte.

\section{Versagerquote für CDC und WHO nicht mehr akzeptabel}

Die Rate der Therapieversager betrug $6,77 \%$, und lag damit über dem akzeptablen Grenzwert von 5\% der CDC und WHO. Das Therapieversagen war je nach Wert des MIC (minimale Hemmkonzentration) unterschiedlich groß. Betrug er bei den Isolaten mindestens $0,12 \mu \mathrm{g} / \mathrm{ml}$, lag die Versagerquote bei $25 \%$ (7 von 28 Patienten). Bei weniger als $0,12 \mu \mathrm{g} / \mathrm{ml}$ lag die Quote nur bei $1,90 \%$ (2 von 105 Patienten). Aus den Ergebnissen pharmakokinetischer und pharmakodynamischer Studien schließen Infektiologen generell, dass Neisserien mit einer MIC von mindestens $0,12 \mu \mathrm{g} / \mathrm{ml}$ bei einer oralen Therapie mit einmal $400 \mathrm{mg}$ Cefixim nicht erfolgreich bekämpft werden können. Deshalb wird dieser Grenzwert auch vom European Committee on Antimicrobial Susceptibility Testing (EUCAST) mit Resistenz gleichgesetzt.

Allen VG et al, JAMA 2013, 309(2):163

\section{Innerhalb von fünf Jahren}

\section{Fast jede vierte Meniskusnaht}

\section{versagt}

\begin{abstract}
Muss der Meniskus entfernt werden, leidet der Knorpel - es droht der Gelenkverschleiß. Verletzte Menisken werden daher möglichst erhalten, Risse genäht. Die kurzfristigen Erfolgsaussichten eines solchen Eingriffs sind relativ gut untersucht, zu den langfristigen gibt es kaum Daten. Die wenigen werteten nun US-amerikanische Orthopäden aus.
\end{abstract}

Die Menisken sind wichtige, aber auch sehr vulnerable Strukturen des Kniegelenks. Hat man früher eingerissene Knorpelscheiben kurzerhand entfernt, steht mittlerweile der Erhalt an oberster Stelle, mit immer ausgefeilteren Methoden. So unterscheidet man heute unter anderem die Inside-out-, die Outside-in- sowie die All-inside-Techniken.

\section{Was bringt der technische Fortschritt?}

Der technische Fortschritt brachte auch - wie zahlreiche Studien belegen - den gewünschten Erfolg, zumindest kurzfristig, also ein bis zwei Jahre nach dem Eingriff. Auf lange Sicht hingegen scheint es etwas anders zu sein, wie US-amerikanische Orthopäden kürzlich in einem Review berichteten: Beinahe jeder vierte re- parierte Meniskus machte innerhalb von fünf Jahren wieder Beschwerden. Jeffrey J. Nepple von der Universitätsklinik in St. Louis und seine Kollegen sichteten das vorhandene Studienmaterial nach Erhebungen, in denen die Versagerquote während fünf Jahre nach dem Eingriff oder länger untersucht worden war. Gerade mal 13 Studien erfüllten die Einschlusskriterien, wobei in jeweils fünf offen operiert bzw. der Meniskusriss mit der Inside-out-Technik genäht wurde, in einer die Outside-in-Technik angewandt und in weiteren drei die Knorpelscheibe mit Ankerstiften repariert wurde.

\section{Häufig erneute Operation}

Der Eingriff wurde als nicht erfolgreich gewertet, wenn die Patienten erneut über klinische Symptome klagten, meist auf- grund mechanischer Beschwerden. Viele, aber nicht alle unterzogen sich wegen eines neuerlichen Meniskusrisses einer weiteren Operation. Egal mit welcher Technik der Meniskus nun repariert wurde, die Versagerquoten innerhalb von fünf Jahren waren mit $22,3 \%$ bis $24,3 \%$ ähnlich. Die Versagerrate scheint mit den Jahren zu steigen, wobei etwa 30\% der neuerlichen Beschwerden nach zwei Jahren aufgetreten sind. Vier Studien machten Angaben zur durchschnittlichen Dauer bis zum Wiederauftreten von Symptomen, die reichten von 2,2 bis 4,2 Jahren. Aussagen zu den neueren All-inside-Techniken waren nicht möglich. Für diese Verfahren gibt es bislang keine Daten zum Langzeiterfolg.

Nepple JJ et al, J Bone Joint Surg Am 2012 (online first) 